\title{
НОВОЕ ПОКОЛЕНИЕ
}

\section{ПРОГРАММИРУЕМЫХ ИСТОЧНИКОВ ПИТАНИЯ МОЩНОСТЬЮ ДО 5 кВт OT TDK-Lambda}

УДК 621.311.6:

$: 621.316 .72$ BAK 05.27 .00

\section{Е.Рабинович}

\author{
Читатели журнала наверняка наслышаны о том, что компания \\ TDK-Lambda уделяет особое внимание разработке \\ программируемых источников питания. Публикации последних \\ лет освещали новые модели серии GENESYS и новую серию Z+. \\ А поскольку в компании не принято хвастаться задумками заранее, \\ многие были заинтригованы: что же будет представлять собой \\ следующее поколение управляемых источников? Сентябрь этого \\ года ознаменовался выходом долгожданной линейки GENESYS+, \\ которая представляет собой результат кропотливой многолетней \\ работы инженеров и других специалистов компании.
}

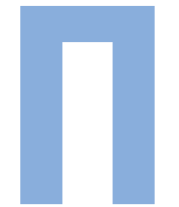

ервые представленные модели имеют мощность 5 кВт. При ширине 19" (483 мм) и высоте стандарта IU это означает непревзойденную для данного типа приборов удельную мощность. В дальнейшем будут производиться модели мощностью 3,4 и 1,7 кВт с выходными напряжениями от 10 до 600 В. Такая дискретность номиналов мощностей указывает на то, что новая серия унаследовала очень эффективную и уже проверенную многолетним опытом концепцию модульной структуры. Благодаря ей конструкция прибора является упорядоченной и надежной, процесс технического обслуживания становится намного эффективнее и проще, а разработка модельного ряда и производственная сборка занимают существенно меньше времени.
Источники питания (ИП) линейки GENESYS+ (рис.1) несут на борту центральный высокопроизводительный контроллер, работающий на частоте 72 МГц, и 168-мегагерцовый DSP-процессор, которые делают новую серию без преувеличения новаторской. Цифровая технология, во-первых, расширяет функциональные возможности, доступные пользователю (о чем мы расскажем подробнее), во-вторых, позволяет обеспечивать более тесную взаимосвязь между отдельными узлами и платами прибора, а в-третьих, позволяет достигать более высоких характеристик.

Модульная концепция не только повлияла на внутреннюю архитектуру устройства, но и обеспечила гибкую платформу для расширения: специальная запатентованная схема межмодульной коммуникации позволит собирать более эффективные 
Модели серии GENESYS+ мощностью 5 кBт

\begin{tabular}{|c|c|c|c|}
\hline Модель & $\begin{array}{c}\text { Выходное } \\
\text { напряжение, } \\
\text { В пост. тока }\end{array}$ & $\begin{array}{c}\text { Выходной ток, } \\
\text { A }\end{array}$ & $\begin{array}{c}\text { Выходная } \\
\text { мощность, } \\
\text { Вт }\end{array}$ \\
\hline \multicolumn{4}{|c|}{ Доступные модели } \\
\hline G10-500 & $0-10$ & $0-500$ & 5000 \\
\hline G20-250 & $0-20$ & $0-250$ & 5000 \\
\hline G30-170 & $0-30$ & $0-170$ & 5100 \\
\hline G300-17 & $0-300$ & $0-17$ & 5100 \\
\hline G600-8.5 & $0-600$ & $0-8,5$ & 5100 \\
\hline \multicolumn{4}{|c|}{ Выход в первом квартале 2018 года } \\
\hline G40-125 & $0-40$ & $0-125$ & 5000 \\
\hline G60-85 & $0-60$ & $0-85$ & 5100 \\
\hline G80-65 & $0-80$ & $0-65$ & 5200 \\
\hline G100-50 & $0-100$ & $0-50$ & 5000 \\
\hline G150-34 & $0-150$ & $0-34$ & 5100 \\
\hline
\end{tabular}

работе значительно выросла и исчезла необходимость задавать количество ведомых звеньев (как в случае с параллельным включением серии GENESYS). Также благодаря данной шине улучшены показатели динамической стабильности выходных параметров при различных возмущающих воздействиях: они лишь незначительно уступают тем же показателям отдельно работающего ИП. При этом вся система работает как одно целое, а в случае неполадки в одном из блоков выдается сигнал ошибки, что препятствует перегрузке и выходу из строя остальных звеньев. Проведенные на данный момент испытания распространяются на четыре модуля, соединенных параллельно, но цифровое управление позволит увеличивать количество параллельно работающих блоков.

Также для удобства пользователей будут доступны готовые сборки мощностью 10 и 15 кВт, состоящие из одного ведущего модуля, через который осуществляется всё управление, и одного или двух ведомых, не имеющих органов управления с передней панели (рис.2). Это позволит оптимизировать денежные затраты

и стабильные системы, состоящие из параллельно работающих блоков. Новая внутрисистемная информационная шина позволяет ведущему модулю постоянно следить за количеством рабочих силовых модулей в системе, поэтому точность распределения токов нагрузки между всеми иП при параллельной

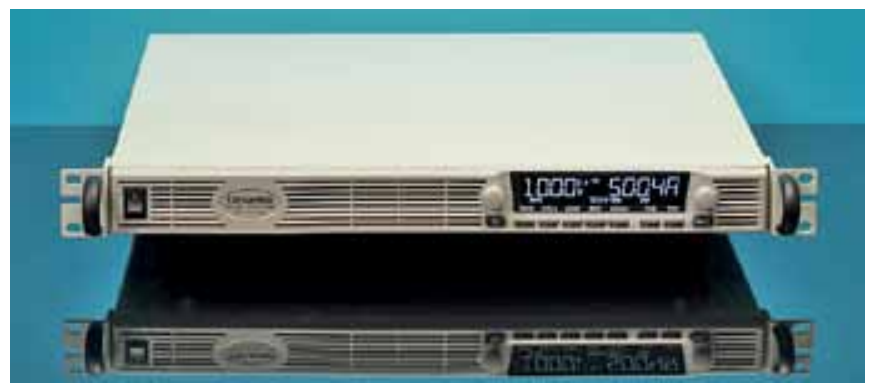

Рис.1. Внешний вид программируемого источника питания серии GENESYS+ при наборе более мощных систем.

Поскольку топология источников питания претерпела существенные изменения, КПД данной серии существенно превышает параметры GENESYS 5 кВт и составляет 90-92\% по сравнению с 84-88\% предшественника. При этом весить новое устройство будет менее 7 кг, что в два раза меньше веса его более раннего аналога.

ИП GENESYS+ работают не только в режиме стабилизации тока и напряжения, но и в режиме
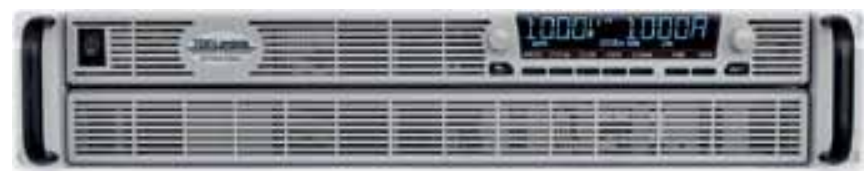

Рис.2. Готовая сборка из двух источников питания серии GENESYS+ мощностью $10 \mathrm{kBT}$ 


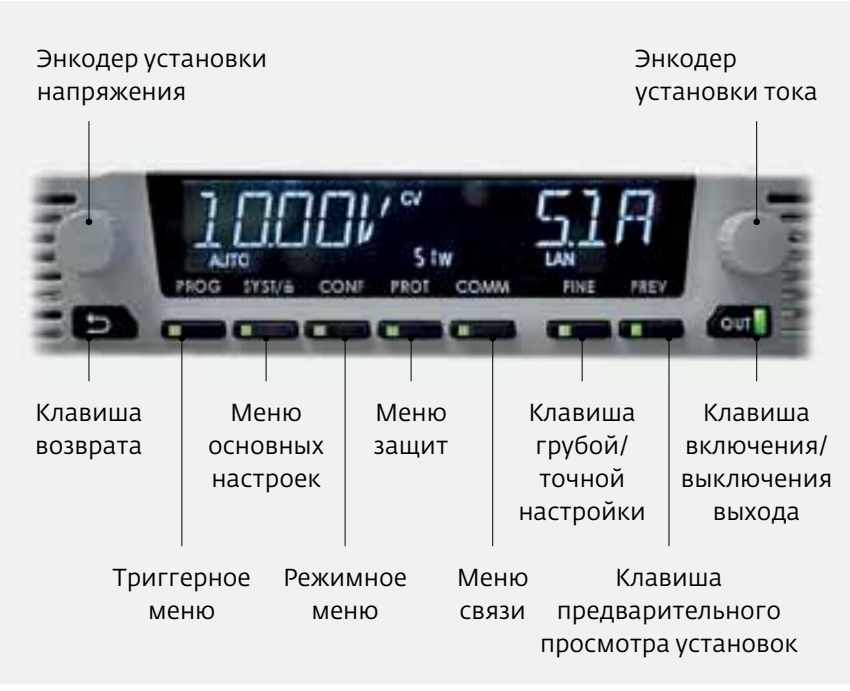

Рис.3. Вид панели управления программируемого ИП серии GENESYS+

постоянной мощности (Constant Power Mode). Для реализации данной функции пользователь может задать значение ограничения выходной мощности и в дальнейшем при изменении выходных показателей тока (напряжения) процессор будет подстраивать выходные значения напряжения (тока) по закону постоянной мощности.

Среди новых функций хочется отметить возможность установки произвольного времени спада/ нарастания сигнала тока или напряжения (Slew Rate Control). Настройка данной функции доступна как при управлении с передней панели, так и через интерфейс, а ее реализация осуществляется простым заданием значения изменения выходного сигнала за одну миллисекунду. Пользователь может задать все эти четыре параметра по отдельности -для нарастания и спада тока и для нарастания и спада напряжения. В качестве примечания, важно отметить, что скорость спада/нарастания не может быть больше, чем физические возможности, отмеченные в спецификации.

При заказе базовой версии любой модели пользователи получат в свое распоряжение не только изолированный аналоговый порт и цифровой интерфейс RS232/485, но также USB2.0 и сетевой порт LAN (LXI 1.5). При этом новая серия полностью совместима с линейками GENESYS и Z+, так как программное управление реализовано с помощью тех же командных языков (GEN и SCPI). В качестве опциональных интерфейсов можно будет вооружиться шиной GPIB, а также интерфейсной платформой Anybus, которая в будущем будет поддерживать шины типа Modbus, PROFIBUS, DeviceNet, EtherCAT и др.
При том, что новое изделие унаследовало все ключевые элементы и цветовую гамму серии GENESYS, изменения не смогли не коснуться внешнего вида прибора. Прежде всего это новый жидкокристаллический дисплей передней панели, который обладает высокой контрастностью и широким углом считывания показаний. В отличие от предшественников, он синего цвета и выдает индикацию с помощью 16 сегментов при одновременной буквенной индикации всех основных режимов работы и настроек параметров.

Все настройки ИП G+, как и в серии Z+, задаются с помощью системы меню настроек. Но в отличие от упомянутой серии здесь предусмотрены не три, а пять главных меню: меню основных настроек (системное меню), меню связи, меню защит, режимное меню и триггерное меню. Вход в эти меню настроек при ручном управлении осуществляется нажатием на клавиши передней панели: "Syst", "Comm", "Prot", "Conf" и "Prog" соответственно (рис.3). В каждом из этих разделов с помощью энкодеров двойного действия выбирается нужный подраздел и осуществляется выбор параметра и ввод его значения.

Системное меню позволяет выбирать вид управления (местное или удаленное), работать с выбором ячеек памяти для записи или вызова произвольных функций, осуществлять переход между местной и удаленной обратной связью, включать или выключать схемy Preload, настраивать работу дисплея, а также настраивать несколько других функций.

Меню связи предназначено для выбора вида активного интерфейса, задания порядкового адреса источника, установки скорости обмена данными, установки IР-адреса и МАС-адреса при работе с LAN-интерфейсом, а также выбора языка программирования.

Меню защит позволяет задать уровень защиты по перенапряжению, установить или отключить защиту типа Foldback (отключение выхода при достижении границы установленного тока или напряжения), установить уровень защиты по низкому напряжению, а также задать значения задержек срабатывания защиты по низкому напряжению и защиты Foldback.

В режимном меню включаются режимы "Start" или "AutoRestart", выбирается вид программирования, диапазоны аналогового управления, настраиваются режим ограничения мощности и вводятся параметры функции Slew Rate Control.

Триггерное меню настраивает работу входных и выходных триггеров при задании произвольных форм сигнала и при необходимости позволяет установить их задержки. 

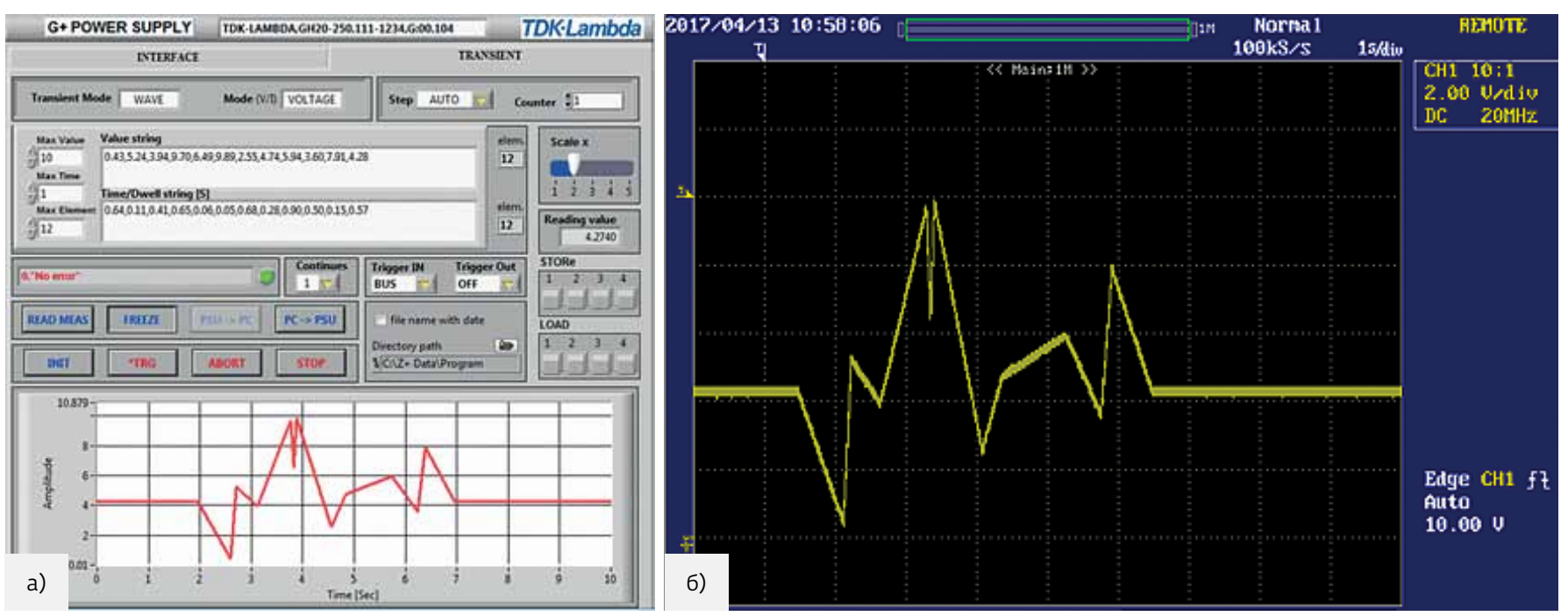

Рис.4. Возможность задания произвольных функций: а - окно графического интерфейса, б - осциллограмма, снятая с выхода ИП

Одной из главных отличительных особенностей является возможность задания произвольных форм выходных сигналов напряжения и тока (рис.4а, б). Настройки задания таких функций осуществляются программно посредством команд или с помощьюграфических интерфейсов, разработанных производителем (рис.4а) и доступных в разделе лабораторных источников питания на сайте: www.tdk-lambda.ru или через запрос по адресу info@tdk-lambda.ru. Принцип задания остается тем же: создается последовательный список значений тока или напряжения, а также список временных значений, соответствующих необходимому времени задержки сигнала на одном уровне или времени перехода на следующий уровень. Также возможна установка дополнительных параметров, например значения количества циклов, которое обеспечивает повторение одной функции необходимое количество раз (на рис.4 этот параметр равен "1").

Настройки любой заданной формы можно занести в память и в дальнейшем вывести без необходимости повторного задания всех параметров и списков. Для этого отведены четыре ячейки памяти. Но, в отличие от модуля Z+, обладающего подобной функцией, максимальное количество точек произвольной формы увеличено с 12 до 100.

Дополнительная нагрузочная плата (Preload), благодаря которой время спада напряжения становится более коротким, у новых приборов стала отключаемой. Это еще одно преимущество, позволяющее приборам более безопасно работать в областях применения, связанных с аккумуляторными батареями, когда через такую плату может происходить непроизвольный разряд накопленной энергии

Несомненно, GENESYS+ обладает и целым рядом уже привычных функций, таких как:

- запоминание последних настроек (Last Setting Memory);

- функции защит от перенапряжения и от перегрузки по току;

- защита типа FoldBack;

- защита от перегрева;

- выбор режима автоматического перезапуска (AutoRestart) или безопасного перезапуска (Safe Start);

- выносная обратная связь;

- поддержка соединения Multi-Drop

и многие другие функции.

В данной статье подробно описаны наиболее яркие функциональные отличия новой серии, чтобы предоставить читателю ясную картину о новом мощном и гибком инструменте, который с успехом найдет свое применение не только в уже знакомых нам промышленных процессах и научных установках, но и в решении новых задач, о которых мы надеемся узнать в ближайшем будущем.

Все модели GENESYS+ имеют гарантию пять лет, отвечают стандартам IEC/EN61204 по кондуктивной и излучаемой электромагнитной совместимости для условий промышленного применения (FCC, часть 15-А), а также одобрены в соответствии со стандартами безопасности UL/EN/IEC60950-1. Более подробную информацию о новых источниках питания можно найти на сайте www.tdk-lambda.ru в разделе программируемых источников питания. 